\title{
La observación del problema ambiental en un contexto de diferenciación funcional
}

\author{
The Observation of the Environmental Problem in the Context of Functional Differentiation
}

Anahí Urquiza

Departamento de Antropología; Centro del Clima y la Resiliencia (Cr2); Núcleo Interdisciplinario de

Estudios Socioambientales, Universidad de Chile

\section{Bárbara Morales}

Centro del Clima y la Resiliencia (Cr2); Núcleo Interdisciplinario de Estudios Socioambientales, Universidad de Chile

RESUMEN

El presente trabajo es fruto de una investigación social cualitativa realizada el año 2014 en el marco del Núcleo Interdisciplinario de Estudios Socioambientales de la Universidad de Chile (NIES) y con el apoyo del Centro de Ciencia del Clima y Resiliencia (CR2). La investigación se centra en el problema ambiental desde tres ámbitos sociales: público, privado y conocimiento experto. El estudio identificó la existencia de tres tipos de obstáculos que dificultarían el diálogo entre los actores que son parte de la discusión, así como el tratamiento del problema socioambiental, estos son: obstáculos a nivel de institucionalidad y normativa vigente; obstáculos a nivel del actuar y de las relaciones entre los actores; y obstáculos que se presentan a nivel de los significados asignados al problema. En el presente artículo se abordan los últimos dos tipos de obstáculos desde una perspectiva sistémico constructivista.

PALABRAS ClaVE: Problemas socioambientales; Teoría de sistemas sociales; Investigación cualitativa; Políticas ambientales chilenas; Conflictos socioambientales

\section{ABSTRACT}

This paper is the result of a qualitative research conducted in 2014 under the Interdisciplinary Center for Social and Environmental Studies at the University of Chile (NIES) and with the support of Center for Climate and Resilience Research (CR2). The research focused on the environmental problem from three social areas: public, private and expert knowledge. Three types of obstacles are identified, which difficult both the dialogue between actors and the addressing of socio-environmental problem, i.e. obstacles at the level of institutions and regulations; obstacles and actions at level of relations between actors; and obstacles that arise of the meanings assigned to the problem. In this paper the last two types are addressed from a systemic-constructivist perspective.

KEYWORDS: Socio-environmental problems; Social systems theory; Qualitative research; Chilean environmental policies; Environmental conflicts

\section{INTRODUCCIÓN}

En una sociedad funcionalmente diferenciada los problemas socioambientales son tematizados desde múltiples perspectivas, lo que contribuye a su complejización debido a las diferentes racionalidades que se contraponen

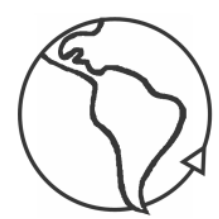

REVISTA MAD - UNIVERSIDAD DE CHILE

Revista del Magíster en Análisis Sistémico Aplicado a la Sociedad

Facultad de Ciencias Sociales, Departamento de Antropología

Universidad de Chile

ISSN 0718-0527

www.revistamad.uchile.cl 
al abordarlos. En el presente artículo se problematizan las descripciones que se realizan desde algunas de las principales perspectivas involucradas al tematizar los problemas socioambientales.

El análisis que se presenta a continuación es fruto de un estudio realizado el año 2014 en el marco del trabajo del Núcleo Interdisciplinario de Estudios Socioambientales de la Universidad de Chile (NIES) y con el apoyo del Centro de Ciencia del Clima y Resiliencia (CR2) ${ }^{1}$. La metodología utilizada fue de carácter cualitativo, contemplando la realización de veintiocho entrevistas semi-estructuradas y de un grupo de discusión. La muestra, por su parte, fue de carácter estructural, y consideró los distintos ámbitos desde los cuales actualmente se discute el problema socioambiental en Chile. Así, en el ámbito público, fue considerada la mirada de parlamentarios, representantes de gobierno, funcionarios públicos y municipales. En el ámbito del conocimiento experto, fue considerado el punto de vista tanto de académicos vinculados a la temática como de profesionales que actualmente son parte de consultoras especializadas en el tema. Finalmente, dentro del ámbito privado fue considerado el discurso tanto de empresarios como de trabajadores.

En cuanto a sus resultados, el estudio identificó la existencia de tres tipos de obstáculos que dificultarían tanto el diálogo entre los actores como el tratamiento del problema socioambiental, estos son: obstáculos a nivel de institucionalidad y normativa vigente; obstáculos a nivel del actuar y de las relaciones entre los actores; y obstáculos que se presentan a nivel de los significados asignados al problema. En el presente artículo se abordan los últimos dos tipos desde una perspectiva sistémico constructivista, problematizando la tematización de lo socioambiental desde la policontexturalidad societal moderna.

En el primer apartado se abordan algunas cuestiones básicas desde la perspectiva sistémico-constructivista, concentrándonos en la diferenciación funcional y las relaciones entre sistemas observadores. Posteriormente se abordan las descripciones relacionadas con el ámbito público, privado y de conocimiento experto, analizando las autorreferencias y heterorreferencias de cada una de las descripciones. Finalmente se identifican las principales distinciones utilizadas desde cada una de estas perspectivas y se señalan algunas conclusiones de la experiencia presentada.

${ }^{1}$ Proyecto FONDAP 15110009 
1. COMUNICACIÓN ECOLÓGICA EN LA SOCIEDAD FUNCIONALMENTE DIFERENCIADA

La sociedad contemporánea es definida por Niklas Luhmann como una sociedad funcionalmente diferenciada $(1984,1997)$, en otras palabras, caracterizada por la diferenciación interna en múltiples sistemas que se orientan a la resolución de problemas específicos. Desde esta perspectiva, cada sistema cumple una función y está limitado por sus propias estructuras, lo que implica que éstos sólo tienen la posibilidad de orientarse internamente hacia problemas que ellos mismos identifican como relevantes y en cada uno de ellos conecta la comunicación que se genera bajo sus propias limitaciones. De esta forma, en el sistema político sólo conecta comunicación política, sin posibilidad de enlazar, por ejemplo, comunicación religiosa a menos que sea observada políticamente, la comunicación económica no conecta con comunicación moral, ni tampoco la comunicación científica con comunicación política, y así para cada uno de ellos. En este contexto, la comunicación ambiental -o ecológica- sólo logra irritar a los sistemas que tienen la posibilidad de observarla desde sus propias estructuras, limitando la posibilidad de resonancia de estas comunicaciones y complejizando el tratamiento global de los efectos no esperados de las operaciones sociales en el medio ambiente.

De manera paralela, se encuentran los sistemas organizacionales, que operan a través de decisiones, definen sus membrecías internas y se orientan al cumplimiento de objetivos. Este tipo de sistemas sociales se desarrolla de forma paralela y complementaria a los sistemas funcionales, especializando aún más la comunicación y complejizando el tratamiento de los problemas ambientales. Es en las organizaciones donde se desarrolla la comunicación especializada para el cumplimiento de fines, lo que reduce la complejidad social al limitar las posibilidades de enlace, pero aumentando la complejidad general al desarrollar una mayor especialización en la comunicación (Luhmann 2000). Así, las empresas generan comunicación económica pero relacionada a los servicios o bienes que ofrecen, los partidos políticos procesan la comunicación política a partir de su posición en el gobierno o la oposición, y la comunicación religiosa está determinada por las estructuras de las iglesias y comunidades que la generan. Asimismo, al tematizar los problemas ambientales, cada organización está limitada por sus propias estructuras, distinguiendo sólo lo que puede distinguir a partir de sus estructuras de observación. De esta forma, organizaciones privadas, organizaciones públicas y organizaciones no gu- 
bernamentales observan los problemas ambientales a través de sus propias estructuras, siendo sus objetivos organizacionales los que estructuran la comunicación y complejizan, a su vez, la comunicación societal.

Cada subsistema -tanto sistemas funcionales como sistemas organizacionales- está clausurado operativamente y observa su entorno a partir de las distinciones que tiene disponibles, en otras palabras, es ciego a las selecciones de sentido que no son observables con sus propias estructuras, lo que aumenta la dificultad de coordinación entre los diferentes observadores.

La consecuencia de esta diferenciación interna es una sociedad que no posee un centro o cúspide que otorgue orden o coherencia a la sociedad en su conjunto y cada uno de sus subsistemas. Esta sociedad funcionalmente diferenciada experimenta, de este modo, un policentrismo crónico, que impide controlar desde un vértice el resto de la sociedad y desde allí coordinar una sola mirada sobre el entorno ambiental. Por el contrario, existen múltiples subsistemas que se influyen mutuamente y cada uno observa el entorno ambiental o ecológico desde su propia posición (y sus propias limitaciones).

En este contexto, se vuelve relevante considerar el concepto de "racionalidad", entendiéndola como la unidad de las distinciones utilizadas en la observación, encontrando la racionalidad de cada sistema en el punto ciego de la observación (donde la observación implica la diferenciación entre sistema y entorno). Bajo este marco es evidente entonces, que existen tantas racionalidades como observadores posibles, no hay sólo una racionalidad. Asimismo, los problemas ambientales sólo pueden ser abordados desde las estructuras existentes en la comunicación social, pues éstos existen solamente en la medida en que se comunica sobre ellos (Luhmann 1986).

Cabe señalar algunos ejemplos. El sistema económico puede reducir el medio ambiente a costos y precios o incluso podríamos hablar de una mercantilización de la naturaleza (ej. Turismo). Para el sistema jurídico el medio ambiente puede aparecer a través de normativas, recursos judiciales o juicios (judicialización de los conflictos). Por su parte, el sistema científico puede identificar problemas ambientales y generar conocimiento en torno a ellos, mientras que en el sistema político la comunicación ambiental puede transformarse en votos o en problemas de popularidad. El entorno ambiental -o ecológico- sólo logra ser tematizado por alguno de los sistemas sociales cuando desde las limitaciones estructurales de estos sistemas es posible comunicar sobre ello. Si los problemas ambientales 
son relevantes para los votantes, estos se transforman en un tema para los partidos políticos, mientras que si los consumidores incorporan el potencial impacto de los productos que consumen en sus decisiones, reducir el impacto ambiental pasará a ser un tema relevante para las empresas involucradas.

Cada uno de estos puntos de observación tiene sus propias limitaciones (o puntos ciegos) y aunque es evidente que hay algunos sistemas más influyentes que otros (no podemos obviar la gran resonancia de la economía o la política), ninguno de ellos tiene la posibilidad de dirigir al resto. Una de las consecuencias más evidentes de esto, es que cuando enfrentamos un problema ambiental o hay disputas por los recursos naturales, cada subsistema observa desde sus propias estructuras y genera comunicación desde sus propias particularidades (papers, negocios, procesos judiciales, discusión política, etc.). Estas condiciones son un síntoma inequívoco del policentrismo social, que sin duda se presenta como un gran desafío para generar acuerdos y enfrentar de forma conjunta los problemas socioambientales de la actualidad.

Desde esta perspectiva, también se asume que la comprensión de lo ambiental está limitada por las estructuras del observador, por sus límites de tolerancia y por la aceptabilidad de los riesgos (Douglas 1996), haciendo evidente la necesidad de reflexionar sobre las posibilidades de observación y la relación que se establece entre los diferentes observadores que son parte de los problemas ambientales.

Uno de los efectos no deseados de esta multiplicidad de miradas, de este policentrismo social, es que al enfrentar problemas ambientales, las diferentes perspectivas se contraponen, dificultando una reacción social coordinada que permita hacerles frente. Así, es posible observar cómo las imputaciones de causas y efectos de los problemas ambientales se desplazan a través de sistemas autónomos, generando comunicaciones contradictorias que impiden una reflexión unitaria que permita abordar coordinadamente problemas globales. En algunos casos, incluso, esta multiplicidad de perspectivas se traduce en la multiplicación o amplificación de los efectos de los problemas ambientales.

En este contexto, es posible hablar de "racionalidad reflexiva" de un sistema, cuando este es capaz de observar su propia observación, dicho en los términos del matemático británico George Spencer-Brown (1979), cuando se puede reingresar en el sistema la distinción en la distinción o cuando la re-entrada de la forma en la forma permite observar el punto ciego de la distinción, desplegando la paradoja de la racionalidad (opera- 
ción que demanda tiempo y autoorientación en las distinciones). A partir de este proceso, un sistema puede construir su propia complejidad y, por tanto, su irritabilidad. Puede complementar la distinción sistema/entorno y aumentar sus posibilidades de observación, reaccionando a la frecuencia de sus irritaciones. La reentrada de la distinción en lo ya distinguido enriquece al sistema, generando capacidades de enlace más complejas. Este proceso no se trata, sin embargo, de un ideal, es decir, que esto ocurra no implica que se resolverán los problemas de coordinación entre los sistemas, pues no existe la posibilidad de generar una racionalidad unitaria. Dicho en otros términos, ningún subsistema tiene la posibilidad de reflexionar dentro de sí a la sociedad en su conjunto.

Finalmente, es importante decir que la viabilidad de los sistemas observadores se encuentra estrechamente vinculada a su capacidad de aprendizaje, que permite modificar la identidad del sistema observando sus propios límites y considerar el entorno como constitutivo del sistema. Esta capacidad probabiliza la mantención de sus adaptaciones en la medida en que favorece su coordinación con otros sistemas. En este contexto, los subsistemas sociales tendrían que ser capaces de observar sus propios límites y reconocer su interdependencia recíproca. Esto, a partir de la reflexión, procedimiento que tiene un sistema para re-introducir la diferencia que mantiene con su exterior y sólo a partir de ello enfrentar los desafíos de coordinación que presentan los problemas socioambientales.

A continuación describiremos las observaciones realizadas en torno a los problemas ambientales relacionadas con el sistema político, jurídico, científico y económico, en el contexto de sus principales organizaciones, con el fin de identificar las principales distinciones utilizadas y problematizar posteriormente las diferentes posiciones de observación en el contexto de problemas ambientales.

2. ÁMBITO PÚBlico: COMUNICACIÓN POLÍTICA Y JURÍDICA DESDE LAS ORGANIZACIONES DEL ESTADO

Entre los actores relevantes que se relacionan con los problemas ambientales, se identifican aquellos vinculados al diseño, ejecución y fiscalización de las reglamentaciones ambientales y políticas públicas. Entre estos actores domina la comunicación política y jurídica. Para esta investigación, consideramos la mirada de parlamentarios, representantes de gobierno, funcionarios públicos y municipales. 


\subsection{OBSERVACIONES DE LAS AUTORIDADES SOBRE EL PROBLEMA AMBIENTAL}

Para las autoridades ambientales (representantes del actual gobierno), el problema socioambiental se presenta en mayor medida como un problema técnico que se origina y resuelve en el marco de la institucionalidad ambiental vigente. Partiendo de esta base, son dos las manifestaciones a partir de las cuales el problema toma forma en sus relatos. En primer lugar, por la existencia de zonas altamente contaminadas, muchas de ellas como "pasivos ambientales", cuyo origen sería anterior a la creación y entrada en vigencia de la institucionalidad ambiental.

Simplemente es el pasado lo que nos causa los problemas actuales. Y el aumento de información ambiental nos muestra problemas que no conocíamos, pero no significa que ahora aparecieron. Siempre estuvieron, simplemente ahora nos hacemos cargo de ello y hay que hacer un equilibrio muy claro de demostrar que en realidad no es que las cosas estén peores, simplemente estamos haciéndonos cargo de problemas que antes ignorábamos. (Autoridad, Ministerio del Medioambiente).

En este sentido, las autoridades reconocen que el Estado mantiene una "deuda ambiental" con determinadas zonas del país, que tendría una estrecha relación con la manera desigual con la que se ha proyectado el desarrollo económico. En segundo lugar, el problema toma forma de la mano de los "conflictos socioambientales", que más que ser observados como una reacción de parte las comunidades (afectadas por un determinado problema ambiental), serían, a juicio de los actores, resultado de la desconfianza que aún en la actualidad impera respecto de la institucionalidad ambiental. Se trataría entonces de una crisis de la institucionalidad que tendría su origen en el mal uso que en el pasado se habría hecho de las herramientas institucionales disponibles, específicamente del sistema de evaluación de impacto ambiental (SEIA).

No obstante la falta de confianza, para las autoridades ambientales ambas manifestaciones del problema debiera resolverse en el marco de la institucionalidad ambiental vigente. Pese a que reconocen que aún falta, sobre todo en términos de fortalecimiento de las instituciones, y que aún hay vacíos por enfrentar (como por ejemplo, la ausencia de normativa en materia de conservación de biodiversidad), existe un convencimiento de que en la actualidad la institucionalidad ambiental posee la normativa y los instrumentos de gestión necesarios para hacerse cargo tanto de los pasivos ambientales como de la demanda ciudadana. 
Hacerse cargo del problema implicaría, en estos términos, lograr que la gente vuelva a creer en la institucionalidad ambiental, lo que desde su punto de vista sólo podría lograrse "volviendo al cauce institucional", respetando los procesos existentes, estableciendo "reglas claras", normando la máxima cantidad de procesos y abogando porque lo técnico tenga un peso cada vez mayor a la hora de tomar decisiones políticas. Implicaría, además, hacerse cargo de los focos de contaminación que preceden a la institucionalidad ambiental ${ }^{2}$, muchos de los cuales aún hoy en día seguirían operando fuera de normativa, e incentivar el avance de la sociedad hacia un modelo de consumo más sustentable. Respecto a este último punto, destacan que la preocupación del Ministerio está puesta, por una parte, en el recambio de tecnologías, con prioridad en áreas como transporte y calefacción residencial (donde la tasa de renovación pareciera ser más lenta). Por otra parte, el programa ministerial apunta a la generación de un comportamiento o de prácticas "menos contaminantes" entre quienes habitan los territorios, ámbito en el que la participación de los ciudadanos cobra especial protagonismo. Esto, considerando no sólo que a mayor desarrollo económico del país, mayor será la capacidad de consumo que tendrá la población, sino también que las expectativas de desarrollo que esta última genera (acceso a un auto, a una antena de celular, a mejor calefacción, a aire acondicionado), a su juicio, muchas veces son incompatibles con el cuidado del medioambiente.

\subsection{OBSERVACIONES CRÍTICAS HACIA LAS AUTORIDADES}

La principal crítica dirigida hacia las autoridades, es la falta de voluntad política que existe para enfrentar el problema socioambiental, cuestionamiento que va a desarrollarse a partir de distintos argumentos en el relato de los actores. Expertos, académicos y trabajadores asocian esta falta de voluntad con la tendencia de tratar el problema socioambiental desde una perspectiva técnica, lo que a su juicio refleja el poco interés de las autoridades por asumir su real magnitud y posicionarlo, en consecuencia, como un "problema país".

ONG y organizaciones ciudadanas están de acuerdo, por su parte, en que la falta de voluntad política que caracteriza el actuar de las autoridades tiene directa relación con la manera en que lo económico está influenciando las decisiones ambientales (y políticas en general), sobre todo

\footnotetext{
${ }^{2}$ Frente a lo cual, los actores destacan que se están llevando a cabo "planes de descontaminación" específicos en las zonas afectadas (Puchuncaví, Ventanas, Huasco, Coronel, entre otras).
} 
en el marco de la evaluación de proyectos. Destacan, en este sentido, la débil posición del Ministerio del Medio Ambiente, la manera en que otros ministerios ejercen influencia, la aparición de mandatos superiores tendientes a facilitar la aprobación de proyectos y los intereses cruzados que existen entre las autoridades políticas y la clase empresarial, muchos de los cuales se hacen públicos a la luz de los conflictos. La mayor preponderancia de los criterios económicos por sobre los políticos (entendidos estos últimos desde la perspectiva del bien común), dejaría entonces a las autoridades un margen de acción muy estrecho para enfrentar el problema con el profesionalismo y la transversalidad que éste requiere.

Los miembros de organizaciones ciudadanas cuestionan, además, la indiferencia que muestran las autoridades públicas frente a los argumentos ciudadanos, en su mayoría sustentados por estudios científicos, en un contexto en el que, a su juicio, son pocas las instancias en que es posible participar de manera efectiva en la discusión. Más que un problema de voluntad política, ellos llegan a la convicción de que existe un "problema con la política" frente al cual la única salida posible es movilizarse y ejercer presión.

\subsection{OBSERVACIONES DE PARLAMENTARIOS SOBRE EL PROBLEMA AMBIENTAL}

Los parlamentarios y ex parlamentarios que fueron considerados en este estudio observan el carácter transversal del problema socioambiental a partir de su propio ámbito de vinculación. Destacan, en este sentido, que el tratamiento de "lo ambiental" no sólo se restringe a la Comisión de Medioambiente, éste también es discutido en otras comisiones que de manera directa o indirecta lo aluden (agricultura, energía, obras públicas, pesca, entre otras). Observan también la transversalidad del problema en las distintas ausencias que, a su juicio, aún persisten en materia medioambiental, entre las que destacan: planificación territorial, conservación de biodiversidad ${ }^{3}$, educación ambiental y participación ${ }^{4}$, así como en la persistencia de ciertos enclaves normativos, como el código de aguas o el régimen de concesiones, en el marco de los cuales se privatizaron recursos naturales estratégicos. Plantean, en este sentido, que el problema po-

\footnotetext{
${ }^{3}$ Cuyo Proyecto de Ley, que establece la creación del Servicio de Biodiversidad y Áreas Protegidas, se encuentra actualmente en discusión en el Congreso.

${ }^{4}$ Esta última, desde la perspectiva de uno de ellos, no sólo a nivel de la participación de los ciudadanos en las decisiones que afectan sus territorios, sino también de las regiones en lo que respecta a su propio desarrollo.
} 
see una "dimensión sistémica" a la que, sin embargo, reconocen, aún no se le ha dado verdadera prioridad en el sentido de elaborar una política ambiental que considere su transversalidad.

En segundo lugar, hay consenso entre los parlamentarios al plantear que el problema socioambiental encuentra su origen en el modelo de desarrollo del país. En esta línea, cuestionan la política económica imperante y su relación poco sustentable con el medioambiente, explícita tanto en la legitimación de un modelo de producción y consumo basado en la sobreexplotación de los recursos naturales como en la poca o nula capacidad de regeneración de los entornos intervenidos y la excesiva contaminación.

Los parlamentarios cuestionan, en tercer lugar, el modelo de decisiones políticas del país, sobre todo por su carácter centralista y la manera en que el criterio económico es privilegiado por sobre el resto de las dimensiones de la sociedad -lo que también vinculan a la excesiva concentración económica y al poder alcanzado por los grandes grupos-, propiciando un contexto en el que por regla general el medioambiente es sacrificado en pos del crecimiento económico.

Te lo digo de una manera más brutal, en la abundancia esta agenda tiene más éxito, en la escasez menos éxito, ¿se entiende?, cuando hay plata, los cisnes de Valdivia, cuando hay escasez, échale adelante con las termoeléctricas a como dé lugar. (Ex Diputado)

La identificación de las posibilidades de posicionar la agenda ambiental solamente cuando hay buenos períodos económicos es fundamental para comprender la mirada de los parlamentarios. Para ellos, el problema ambiental es relevante cuando lo es también para los votantes, por lo que si para el electorado las condiciones económicas son importantes, como ocurre en Chile, posicionar los temas ambientales en la agenda pública se hace aún más difícil cuando hay condiciones económicas complejas (como los períodos de crisis).

En la medida en que son las regiones y los sectores de menores ingresos los que resultan mayormente afectados, los parlamentarios destacan, en cuatro lugar, la relación de los problemas ambientales con otras problemáticas de país, como la centralización y la desigualdad. La existencia de "zonas de sacrificio" aparece, en este sentido, como una de las principales manifestaciones del problema, donde además de las consecuencias que se producen sobre el medioambiente natural (agotamiento de los recursos naturales y pérdida de biodiversidad), los parlamentarios hacen 
hincapié en los efectos que se generan sobre la salud y las condiciones de vida de las personas, visibilizados en gran medida a partir de conflictos socioambientales emblemáticos de los se han hecho parte en los últimos años. Además, cobra protagonismo en sus relatos el agotamiento de los recursos naturales y la degradación del medio natural, vinculados a fenómenos climáticos específicos como el calentamiento global, la desertificación y los problemas de agua que estarían afectando a varias zonas del país.

Por último, y en términos de buscar una solución al problema, entre los parlamentarios existe consenso respecto a la necesidad de introducir regulaciones al modelo de desarrollo. No obstante, dependiendo del sector político que representan, hay diferencias en sus posturas respecto al tipo de reglas que se requieren establecer. Algunos destacan la necesidad de establecer "reglas claras" que permitan poner límites a la manera en que lo económico está influyendo en las decisiones políticas. Otros, en cambio, hacen hincapié en la necesidad de establecer "nuevas reglas del juego", lo que implicaría replantearse el modelo de desarrollo y reformular la arquitectura política del país.

\subsection{OBSERVACIONES CRÍTICAS HACIA LOS PARLAMENTARIOS}

Los propios parlamentarios y ex parlamentarios son quienes identifican los principales obstáculos que atraviesan su gestión. En esta línea, el principal problema se presentaría al momento de legislar, de votar las leyes. Es ahí donde vuelven a tener protagonismo las diferencias ideológicas y las influencias de las zonas a las que representan, lo que se traduce generalmente en la reticencia de una buena parte de los parlamentarios a entregar mayores atribuciones al Estado en términos de fiscalización, dotación de recursos y mayor capacidad de personal. Los actores destacan que si bien en el discurso todos están de acuerdo en pedir mayor sustentabilidad, son pocos los parlamentarios que se muestran proclives a "más Estado" para avanzar en ese camino al momento de legislar.

A lo anterior se suma el poco conocimiento y entendimiento que muestran los parlamentarios respecto de la temática, tanto desde el punto de vista de los problemas ambientales como del diseño institucional crítica que también alcanza a los asesores. Algunos actores vinculan esta ausencia de conocimiento a la existencia de una brecha generacional a partir de la cual las antiguas generaciones estarían aún muy cercanas a una lógica productivista en la que el medioambiente sigue siendo obser- 
vado en función del crecimiento económico. Otros, en cambio, creen que la falta de conocimiento es más bien una muestra de la poca importancia que aún en la actualidad, y más allá del discurso, los parlamentarios asignan al problema.

2.5. OBSERVACIONES DE FUNCIONARIOS PÚBLICOS Y MUNICIPALES SOBRE EL PROBLEMA AMBIENTAL

"Dar cumplimiento a la norma" es tal vez el principal criterio a partir del cual los funcionarios públicos observan el problema socioambiental. Es la ley la que determina los parámetros de su existencia así como las competencias y facultades de los distintos servicios en relación con su tratamiento (sea en términos de protección, prevención, conservación, educación o participación).

Asimismo, las escalas o manifestaciones del problema van a tener directa relación con los ámbitos en los que se desenvuelve la gestión de los funcionarios. Éste puede aparecer bajo la forma de la contaminación atmosférica, las zonas de sacrificio, la existencia de vertederos ilegales, el cambio climático o por los conflictos que se generan entre comunidades y empresas cuando ingresa al sistema de evaluación un proyecto de envergadura, manifestaciones que dan cuenta, a su vez, de las preocupaciones actuales del Ministerio en materia medioambiental. Existe consenso, sin embargo, al afirmar que no todas poseen la misma prioridad. El problema es observado, en estos términos, como un problema técnico que se define y resuelve dentro del marco legal.

Ahora bien, al afirmar que aún existen vacíos en materias como ordenamiento territorial, energía o educación ambiental, que requieren una discusión más ampliada a nivel país y la generación de política pública para su tratamiento, los funcionarios públicos salen del ámbito meramente técnico de observación del problema. Lo hacen también al establecer un vínculo con temas sociales y políticos, como la desigualdad, que a su juicio no han sido debidamente considerados en la discusión, convicción a la que llegan de la mano de su relación cotidiana con otros ministerios y servicios con competencia ambiental, así como por los vínculos que establecen con municipios y comunidades locales, a las que van dirigidas buena parte de las políticas de prevención, educación y sensibilización. En este sentido, ellos reconocen que si bien el problema socioambiental "no respeta fronteras" y puede afectar de igual manera a todos los habitantes de una región, sobre todo bajo la forma de la contaminación, son las zonas y 
habitantes con menores recursos quienes viven más de cerca sus consecuencias.

Los funcionarios municipales, en cambio, observan el problema socioambiental sobre todo como un problema cotidiano, cotidianeidad que dice relación con las distintas manifestaciones que éste encuentra y en las que está focalizada, en gran medida, la gestión ambiental local.

Un primer aspecto en el que se manifiesta el problema, es la contaminación que se genera como consecuencia de las malas prácticas que se observan entre los vecinos en el marco de su dinámica cotidiana con el entorno, donde destacan: la quema de basura, la tala de arboles, la existencia de micro basurales; o prácticas mucho más comunes pero que igualmente pueden causar impacto, como la tenencia de mascotas. A juicio de los funcionarios, estas problemáticas tienen directa relación con el desconocimiento que existe entre los vecinos respecto de su medioambiente local, lo cual se ve agravado por la poca relevancia que encuentra la temática en el marco de la gestión municipal. Destacan que hacer frente al problema ha requerido, en este contexto, poner en marcha un proceso educativo tendiente a posicionar la temática en el contexto local (no sólo a nivel escolar sino también a nivel de las organizaciones sociales y los vecinos en general $)^{5} \mathrm{y}$ a introducir a los vecinos en prácticas ambientales más sustentables, donde la sustentabilidad apunta no sólo a interactuar de manera distinta con el medioambiente, sino también a promover la generación de consensos entre los distintos actores que ocupan el territorio con miras a promover un sentido de comunidad.

El segundo aspecto en el que se manifiesta el problema, es la contaminación generada por la actividad industrial de las empresas que funcionan en las comunas, sobre todo las grandes y medianas ${ }^{6}$, así como los impactos generados sobre el medioambiente y la calidad de vida de los vecinos. En esta línea, las manifestaciones del problema son también variadas y, en no pocos casos, de alto impacto, yendo desde la contaminación por ruidos molestos o malos olores hasta aquella que se produce por el exceso de material particulado, la contaminación por desechos tóxicos o la existencia de terrenos con alta presencia de plomo. En este punto, las ausencias de normas y leyes específicas en materia de biodiversidad y ordenamiento territorial (que en el plano local toma forma de la mano de la ausencia o cambios realizados al plan regulador), se presentan como un

\footnotetext{
${ }^{5}$ Lo que está estrechamente vinculado a las metas que el Ministerio les pide cumplir en el marco de los procesos de certificación ambiental.

${ }^{6}$ Ambas comunas de la Región Metropolitana que participaron en el estudio poseen carácter industrial.
} 
obstáculo especialmente problemático para los funcionarios municipales, no sólo porque hay problemas que quedan sin fiscalizar, por la ausencia de una norma que así lo dicte, sino también porque a veces es la misma ausencia de norma la que puede generar el problema.

Los efectos sobre el medioambiente, la salud y la calidad de vida son, por su parte, el principal argumento de los vecinos para presentar denuncias en el municipio e involucrarse en los conflictos socioambientales que se han desencadenado con las empresas en los últimos años. Frente a esto, los funcionarios también hacen alusión al problema de la desigualdad, afirmando que son los sectores con más alto índice de pobreza los que resultan mayormente afectados por la actividad industrial.

De esta manera, vemos que la cotidianeidad del problema ambiental se relaciona también con los problemas identificados por los otros actores del mundo público: la ausencia de regulaciones, la desigualdad y el carácter problemático del modelo de desarrollo del país.

\subsection{OBSERVACIONES CRÍTICAS HACIA LOS FUNCIONARIOS PÚBLICOS Y MUNICIPALES}

En general, la mayoría de los actores reconoce que muchos de los problemas que se presentan en el marco del actuar de los funcionarios públicos y municipales están estrechamente vinculados a los problemas que aún presenta la institucionalidad ambiental, sobre todo en lo que refiere a ausencia normas, la falta de recursos o la poca dotación de personal. En este contexto, uno de los problemas que con mayor frecuencia deben enfrentar, es la falta de coordinación entre los distintos organismos con competencia ambiental, lo que se traduce, en la práctica, en una superposición de funciones, una alta burocracia y en tiempos de respuesta en extremo largos para pronunciarse respecto de un determinado tema. Hay consenso, en este sentido, al plantear que la transversalidad con la que en teoría se proyecta el trabajo del Estado en materia medioambiental, en la práctica no se logra materializar.

En el caso particular de los funcionarios municipales, éstos reconocen, además, que instalar la temática ambiental depende en gran medida de la voluntad política de la autoridad de turno, lo que se traduce, en la práctica, en que sean los municipios con más recursos y aquellos que muestran un mayor compromiso, los que han logrado desarrollar con éxito las unidades de medioambiente, en un contexto en el que todavía la creación de la institucionalidad ambiental sigue siendo voluntaria a nivel 
municipal. A juicio de los funcionarios, esto sin duda repercute en la capacidad técnica con la que puedan contar los municipios para hacerse cargo del tema, lo que hace urgente avanzar en términos de asignación de recursos y de capacitación.

\section{3. ÁMBITO PRIVADO: EMPRESARIOS Y TRABAJADORES}

Dentro del mundo económico fue considerado el discurso tanto de empresarios como de trabajadores en la medida en que ambos se muestran como partes interesadas en el acceso y la explotación de los recursos naturales; los primeros con énfasis en el desarrollo productivo y los segundos en la generación de empleo.

\subsection{OBSERVACIONES DE EMPRESARIOS SOBRE EL PROBLEMA AMBIENTAL}

Para los actores del empresariado que fueron incluidos en este estudio, el problema socioambiental es, en primera instancia, una consecuencia natural del modelo de desarrollo económico que se ha venido aplicando en las últimas décadas en el país. En este sentido, ellos parten de la premisa de que toda actividad industrial va a generar impactos en la medida en que los proyectos hacen uso de los territorios y explotan los recursos naturales disponibles. Avanzar hacia un desarrollo sustentable implica, en estos términos, hacer un uso eficiente de los recursos, pero siempre sobre la base que habrá impactos.

Y cuando te digo cuidarlo, no es que no haya impacto sobre los recursos naturales, porque vuelvo a la premisa inicial, la actividad industrial genera impactos ambientales, en general la actividad humana. (Gerente de Medioambiente, Empresa Minera).

Partiendo de esta definición, la necesidad de hacerse cargo del problema socioambiental pareciera estar ocupando un lugar cada vez más prioritario, sobre a todo a nivel de las grandes empresas. Pese a que aún no es posible hablar de una política ambiental generalizada, o por sector, el tratamiento de la temática ha implicado la creación de departamentos especializados, la consideración de un ítem en el presupuesto anual y la puesta en práctica de políticas específicas que guían tanto el diseño como la implementación de los proyectos. Incluso, algunos actores reconocen que incorporar el tema ambiental en la gestión de la empresa es favorable para 
la estrategia de negocios en la medida en que les permite diferenciarse de otras empresas del sector.

Junto al incremento de las exigencias medioambientales, tanto en el ámbito de la normativa nacional como internacional, el aprendizaje adquirido de experiencias de conflicto, de errores y desaciertos cometidos durante la puesta en marcha de un determinado proyecto, aparece en el relato de los actores como una de las principales razones por las cuales las empresas se han hecho cargo del problema, respuesta que describen más bien como una reacción ante la presión que han generado las comunidades, que muchas veces han puesto en jaque la viabilidad de los proyectos.

La situación que tuvo que enfrentar Celulosa Arauco fue de tal magnitud para ellos que les cambió completamente el paradigma del tratamiento ambiental. (Gerente de Medioambiente, Empresa Minera)

Los conflictos socioambientales aparecen así, para ellos, como la principal manifestación del problema, principalmente de su carácter social que, reconocen, constituye la variable que más tardíamente incorporaron.

Siguiendo esta línea, hacerse cargo hoy del problema socioambiental implica para los actores del ámbito empresarial considerar los impactos propiamente ambientales que genera un proyecto (afectación de recursos, pérdida de biodiversidad, alteración de la calidad del aire), pero también, y de manera complementaria, aquellos impactos sociales y económicos que dicen relación con cambios en las condiciones y calidad de vida de las comunidades que habitan los territorios afectados (relocalización, afectación de las formas de trabajo, alteración de los usos del medio local). Implica, además, incorporar de manera temprana a las comunidades en el proyecto, identificando desde su diseño la mayor cantidad de impactos posibles para, desde ahí, establecer las mitigaciones e ingresar el proyecto al sistema de evaluación formal. En estos términos, avanzar hacia un desarrollo más sustentable implicaría no sólo apuntar hacia el uso eficiente de los recursos naturales, sino también conseguir que el desarrollo de los proyectos se genere en un contexto de "paz social". En palabras de uno de los actores "hoy en día es importante lograr un buen nivel de rentabilidad para la compañía pero también para la sociedad".

La necesidad de que se establezcan "reglas claras" por parte del Estado también aparece como un requerimiento importante para hacer frente al problema socioambiental. Reglas claras que dicen relación, en primer lugar, con los problemas que se presentan en el marco de la evaluación de proyectos (SEA): en relación a la claridad de los criterios de evaluación, a 
los tiempos que demora el proceso y a la ambigüedad de ciertas normativas, todo lo cual redunda en incertezas que terminan poniendo en riesgo la viabilidad de los proyectos. En esta línea, los actores también cuestionan el hecho de que el sistema de evaluación este diseñado sólo para hacerse cargo de los impactos ambientales, dejando fuera los impactos sociales que, desde su perspectiva, son los que generan mayor conflicto. Más que normativa, para este último punto, los actores demandan que el Estado comience a observar el problema con una visión más holista.

La noción de "reglas claras" dice relación, en segundo lugar, con la necesidad de que temas de fondo que subyacen al problema comiencen a ser discutidos por la sociedad en su conjunto. Destacan en este sentido, la necesidad de generar discusión, de planificar, respecto a materias como energía y ordenamiento territorial que pueden, en definitiva, entregar al sector empresarial las bases políticas de lo que el país quiere en términos de desarrollo económico: qué proyectos van a ser considerados y cuáles no, dónde podrán ser ejecutados y dónde no, qué recursos van a ser utilizados y cuáles no, para así no esperar que sea la evaluación de un proyecto la instancia en la que se discutan estos temas. Algunos avanzan un poco más lejos y plantean la necesidad de que el país decida cuál es el tipo de desarrollo al que se quiere llegar.

Bueno, pongámonos de acuerdo en cómo lo utilizamos, qué hacemos, sinceremos las cosas, abramos los libros, miremos qué es lo mejor, qué es lo mejor para todos (...) La industria se va a adaptar a los requerimientos de la sociedad, (...) si la sociedad quiere gas, gas le podemos dar, y eso vale tanto, si la sociedad quiere sol, bueno eso tiene sus costos. (Gerente Técnico, Empresa Hidroeléctrica)

\subsection{OBSERVACIONES CRÍTICAS HACIA LOS EMPRESARIOS}

Son los mismos empresarios quienes reconocen que en últimos años la industria ha experimentado un cambio de paradigma respecto al tratamiento del problema socioambiental, el cual, afirman, se ha dado en gran medida como respuesta al aumento de conflictos que han puesto en jaque, no sólo la viabilidad de importantes proyectos, sino también la manera de relacionarse con las comunidades (donde la postura hasta hace no mucho tiempo atrás era "no informar a la comunidad"). El cambio es asociado, en segundo lugar, al incremento de los estándares ambientales que vienen de la mano de normativas y exigencias internacionales que superan por mucho el marco regulatorio nacional, lo cual les permite, a su vez, 
hacer una distinción entre las grandes y medianas empresas, destacando entre las grandes las mineras con filial internacional ${ }^{7}$. En su mayoría reconocen, no obstante, que en cada uno de los niveles (grandes, medianas y pequeñas), aún hay empresas que observan lo ambiental como un mero trámite que deben cumplir con miras a conseguir la resolución de calificación ambiental (RCA).

Desde el resto de los actores, también se reconoce que los empresarios han tenido un cambio de actitud respecto de su comportamiento ambiental, sobre todo en lo que concierne al relacionamiento comunitario. Reconocen además el rol que ha cumplido la presión ciudadana y las normativas internacionales en la generación de este cambio, y hacen también la distinción entre quienes sólo quieren limpiar su imagen y quienes, por el contrario, quieren generar cambios de gestión más profundos. No obstante lo anterior, aún existe entre ellos el convencimiento ${ }^{8}$ de que desde la lógica empresarial el problema socioambiental sigue siendo observado en función de la rentabilidad, contexto en el que gestionar los impactos sociales y ambientales se ha vuelto positivo en la medida que favorece la estrategia de negocios. No así la ausencia de normativa y de "reglas claras", que volverían cada vez más riesgosa la inversión. En este sentido, destacan que las demandas de "reglas claras" y normas más precisas por parte del empresariado siguen respondiendo a su preocupación inicial de sacar adelante los proyectos, maximizando sus beneficios y minimizando sus costos. Para ellos, el empresariado aún carece de una visión de país que es necesario desarrollen para que exista una discusión real del problema.

\subsection{OBSERVACIONES DE TRABAJADORES SOBRE EL PROBLEMA AMBIENTAL}

Los representantes de la CUT que fueron incluidos en el estudio reconocen que la discusión en torno al problema socioambiental recién comienza a desarrollarse en el ámbito sindical, lo que responde en gran medida a la contingencia generada por la situación de energía y el desarrollo de conflictos socioambientales emblemáticos (entre los que destacan Hidroaysén y Freirina). Afirman, en este sentido, que antes "lo ambiental" seguía siendo observado como algo ajeno al ámbito laboral, una "preocupación de otros" que no concernía mayormente a los dirigentes sindicales. Cons-

\footnotetext{
${ }^{7}$ Empresas que, en general, son las que presentan mayor organización y capacidad para hacerse cargo internamente de la temática.

${ }^{8}$ Entre parlamentarios, Ong, expertos, funcionarios públicos y trabajadores.
} 
cientes de que incluso a nivel internacional no está muy claro cómo abordar el tema desde el punto de vista de los trabajadores, hoy está en construcción una política de medioambiente a partir de la cual la CUT espera tener un rol activo en la discusión del problema.

Destacan, por otra parte, que son los trabajadores vinculados al sector energía los más avanzados en la materia y quienes, al mismo tiempo, están marcando la pauta para tratar la temática de una manera más generalizada. En el resto de los sectores, reconocen que aún existe reticencia a incorporar la temática como una preocupación legítima de los trabajadores, en parte por el desconocimiento que existe en torno al problema y la manera en que éste podría llegar a afectarles. También por la posición un tanto ambigua en la que quedan los trabajadores en el marco de un conflicto, donde, explican, es común que ser parte de la empresa y de la comunidad se muestre, sobre todo en los medios, como algo contradictorio.

En este contexto, son dos las manifestaciones que el problema socioambiental encuentra en la discusión actual que se desarrolla en el mundo sindical. Por una parte, los riesgos que corren los trabajadores al estar en contacto con agentes contaminantes que puedan resultar dañinos para la salud (pesticidas, asbesto, etc.), donde el énfasis esta puesto en la necesidad de tener un "trabajo seguro y libre de contaminación". En segundo lugar, el problema toma forma en el marco de la discusión en torno a la generación de empleo y el cuidado del medioambiente, aspectos que, reconocen, son aún difíciles de conciliar sobre todo en un contexto país en el que económicamente se ha dado prioridad a las exportaciones y a los acuerdos comerciales en desmedro del medioambiente, donde la normativa medioambiental es aún muy permisiva, donde no existe política energética y en el que la calidad de empleo es aún muy precaria. No obstante lo anterior, y desde la premisa de que "tiene que haber trabajo", están conscientes que su generación no puede ser a cualquier costo, pues en la medida en que se afecta el medioambiente, se afecta también el lugar donde viven, el lugar donde trabajan, eso va afectando la economía y termina igualmente teniendo repercusiones en la generación de empleo. A su juicio, se hace urgente entonces abrir la discusión a los temas de fondo que subyacen al problema.

La CUT quiere ser parte de la discusión y por eso es que nos hemos ido preparando y empoderando, porque tenemos algo que decir, ah, no se debe medir solamente cuánta energía necesitamos y hagámoslo ya y de qué forma, que sea más barata, si no que digamos cuáles son los impactos sociales, el impacto en la 
sociedad, en el medioambiente, en el trabajo, no todo es sólo costos. (Dirigente sindical, Representante de la CUT)

\subsection{OBSERVACIONES CRÍTICAS HACIA LOS TRABAJADORES}

En el caso de los trabajadores, su rol no fue explícitamente evaluado por el resto de los actores, lo que podría deberse a la vinculación más bien tardía que han tenido con la problemática y al hecho de que su figura tiende a ser fusionada con la de otros actores en el marco de la discusión (en general tienden a ser vinculados tanto a los intereses de las empresas como a las preocupaciones de las comunidades).

En cambio, son los mismos representantes de la CUT quienes destacan que uno de los principales problemas que aún persiste en el sector, es el poco conocimiento que existe entre los trabajadores respecto del tema medioambiental, sobre todo en aquellas empresas menos vinculadas al sector productivo. Reconocen, en este sentido, que enfrentar el problema aún está lejos de constituir una prioridad en el mundo sindical.

\section{4. ÁMBITO CONOCIMIENTO EXPERTO: ACADÉMICOS Y CONSULTORES}

Actores relevantes de la discusión en torno a los problemas ambientales, relacionados con la comunicación científica, son los representantes de conocimiento experto. En este marco, el estudio consideró tanto a académicos como a expertos relacionados con consultoras que prestan asesorías en temas ambientales.

\subsection{OBSERVACIONES DE ACADÉMICOS SOBRE EL PROBLEMA AMBIENTAL}

$\mathrm{Al}$ posicionarse en su propio dominio de trabajo, el problema socioambiental aparece para los académicos como un problema de investigación, que plantea preguntas, genera hipótesis y aporta a la generación de conocimiento. La contingencia con la que éste se presenta, donde los conflictos socioambientales ocupan un rol importante en su visibilización, ha visto incrementarse el conocimiento científico en los últimos años e incluso se han establecido puentes entre distintas disciplinas con miras a la comprensión de su complejidad. En este contexto, son varias las líneas de trabajo desde la que hoy en día la academia se está vinculando con el problema: explotación de los recursos naturales, transformaciones y representaciones del medioambiente, conflictos socioambientales, relaciones con el 
medio, biodiversidad, cambio climático, riesgo y vulnerabilidad, entre otras temáticas, cuya problematización va a variar de disciplina en disciplina y de acuerdo a los énfasis y enfoques teóricos desde los cuales se realiza la observación.

Ahora bien, pese a la diversidad de perspectivas de observación del problema, entre los académicos existe consenso al plantear que su origen se encuentra en el modelo de desarrollo del país, convicción a la que algunos llegan de la mano de sus propias investigaciones, otros por su vinculación política con la problemática. Desde ambas esferas, no obstante, el modelo es puesto en cuestión desde un punto de vista económico, pero también político y social. Identifican, en este sentido, que el problema socioambiental es un problema de país, transversal a los distintos dominios en que se desenvuelve la sociedad.

Partiendo de esta base, los académicos destacan la manera en la que el modelo deja en manos del mercado el acceso y la distribución de los recursos naturales, legitimando un contexto de sobreexplotación y uso intensivo del medioambiente que aparece como factor común a muchos de los problemas ambientales que afectan al país. Asimismo, éstos destacan la debilidad que aún existe en materia de institucionalidad ambiental, lo cual dice relación con las pocas atribuciones del Estado en la materia, la ausencia de regulaciones y la mala calidad de éstas. La concentración de los problemas ambientales en las zonas más vulnerables y en aquellas regiones más alejadas del centro económico-político, aparecen por su parte como las consecuencias más visibles del problema.

En el plano de las soluciones hay dos visiones que destacan entre los académicos que fueron considerados en el estudio. Por una parte, hay quienes plantean la necesidad de establecer un diálogo social profundo con vías a discutir las condiciones en las que se está llevando a cabo el desarrollo, lo que implica replantearse la posición que tiene el medioambiente respecto de la economía pero también hacerse cargo de las ausencias que aún se mantienen en materia de institucionalidad ambiental. Hay quienes, en cambio, van un poco más lejos y plantean la necesidad de cambiar de raíz el sistema económico así como la base neoliberal sobre la que se sostiene.

\subsection{OBSERVACIONES CRÍTICAS HACIA LOS ACADÉMICOS}

Es desde el mismo ámbito académico que surge la crítica hacia la posición ambigua y el distanciamiento que ha mostrado la academia respecto de la 
discusión del problema socioambiental. Esta crítica es compartida por el resto de los actores ${ }^{9}$ que observan que, si bien en términos de investigación es notorio el avance que se ha generado, en términos de incidencia política, en cambio, la academia ha optado por mantenerse al margen de lo que ocurre en el país. Afirman, en este sentido, que su preocupación mayor pareciera estar centrada en la generación de papers y no en ser parte de una discusión más ampliada, lo que ha implicado, por otra parte, que su acercamiento a la temática se dé sólo a nivel técnico, para dar opiniones puntuales o prestar un determinado servicio pero casi nunca para tomar una posición o pronunciarse como institución frente a un determinado proyecto, conflicto o problema ambiental.

La poca generación de conocimiento aplicado es también una crítica que se hace recurrente en el relato de los actores ${ }^{10}$. Éstos cuestionan la tendencia de los académicos a centrarse en el conocimiento teórico, dejando de lado la generación de herramientas aplicadas o publicaciones de divulgación que puedan servir de base a comunidades, ONG y profesionales del mundo aplicado para tener un mejor acercamiento al problema. Funcionarios municipales y miembros de ONG agregan, además, la poca o nula capacidad de la academia de devolver el conocimiento generado.

Si la gente quiere saber, si no para qué te sirve saber dónde anda el gato güiña por ejemplo, para qué te sirve si al final la gente igual lo va a ver, quizás qué es lo que le hagan, es mejor que la gente sepa que es una especie que está en categoría de conservación. (Encargada Medioambiente, Municipio Región Metropolitana).

A juicio de los actores, se hace evidente entonces la necesidad de que la academia vuelva a asumir el rol social y político que le compete y en ese camino no sólo comience a socializar sus investigaciones, sino también a participar de una manera más activa tanto en la discusión del problema como en la generación de los cambios políticos que son necesarios para enfrentarlo.

\subsection{OBSERVACIONES DE EXPERTOS CONSULTORES SOBRE EL PROBLEMA} AMBIENTAL

Parte importante de los expertos-profesionales involucrados en los problemas ambientales están relacionados con consultoras ambientales. Des-

\footnotetext{
${ }_{9}^{9}$ Miembros de ONG, expertos, empresarios, parlamentarios.

${ }^{10}$ Sobre todo entre expertos, consultoras y empresarios.
} 
de estos espacios, se observa el problema socioambiental en el marco de la implementación o puesta en marcha de un determinado proyecto, más específicamente de la mano de los impactos que el proyecto genera o podría generar, donde destaca un área de efectos propiamente ambientales, cuyo tratamiento está contemplado en los procesos formales de evaluación ambiental, y un área en la que se gestionan los impactos sociales y económicos susceptibles de generarse sobre las comunidades directamente afectadas. Para esta última, destacan metodologías de relacionamiento comunitario, de gestión de conflictos, de participación temprana, que si bien presentan ciertos elementos que están estandarizados en el sector, se llevan a cabo al margen del proceso formal de evaluación de proyectos (SEA), con excepción del convenio número 169 de la OIT que comienza a ser aplicado para el caso de las comunidades indígenas ${ }^{11}$. Así, es posible identificar que desde el ámbito de acción de las consultoras, los problemas ambientales tendrán relación con la viabilidad de los proyectos y su desarrollo en el marco de una relación armónica con la comunidad.

O sea, aquí no se trata sólo de aprobar proyectos, sino que es aprobar proyectos y desarrollarlos de manera sustentable en su relación con la comunidad, entonces ese ha sido un proceso que yo te diría que en Chile se ha ido produciendo. Y asimilar la importancia que tiene, hace 15 años atrás esto no era tema, hace 15 años atrás las empresas trataban de no tocar el tema, de no informar a la comunidad, esa era la postura. (Gerente de Medioambiente, Consultora Ambiental).

La generación de un conflicto socioambiental aparece, en este contexto, como la principal manifestación del problema que tendrá lugar en la medida en que la relación empresa-comunidad se quiebre y ésta última reaccione ante los impactos, sobre todo sociales, que no lograron ser debidamente mitigados o minimizados.

En relación a los factores que dan origen al problema, las opiniones son dividas entre los profesionales. En una primera postura, destaca el argumento de quienes ven la emergencia del problema como una consecuencia directa de las fallas que aún presenta la institucionalidad ambiental, donde las críticas se dirigen sobre todo hacia el Sistema de Evaluación Ambiental: la manera en que éste debe hacerse cargo de problemas para los que no fue diseñado, la imposibilidad de hacer modificación a los pro-

\footnotetext{
${ }^{11}$ Comienza a regir en diciembre de 2013 en conjunto con las modificaciones que introduce al sistema la Ley 20.417 (2010) que crea el Ministerio del Medioambiente, el Servicio de Evaluación Ambiental y la Superintendencia del Medioambiente.
} 
yectos, la incertidumbre que existe respecto a los tiempos que debe durar el proceso de evaluación y el poco acompañamiento que se le ha dado al mundo empresarial para comprender a cabalidad sus requerimientos técnicos.

Hoy día el mundo empresarial ha tenido que entenderlo a punta de errores y eso ha sido una pérdida para el país. O sea, una pérdida grande para todos, han sido motivos de conflicto, han sido problemas de contaminación, todo lo malo que tú conoces ha sido producto de eso creo yo. (Gerente de Medioambiente, Consultora Ambiental).

Desde este punto de vista, dar solución al problema implicaría sobre todo un esfuerzo de parte del Estado por mejorar la institucionalidad ambiental vigente, estableciendo "reglas claras" en relación a la evaluación de los proyectos, pero también a la planificación territorial, cuya ausencia aparece como un agravante más del problema.

\subsection{OBSERVACIONES CRÍTICAS HACIA LOS EXPERTOS CONSULTORES}

El principal problema que identifican los expertos vinculados a consultoras, es la diversidad de empresas de este tipo que hoy en día están dedicadas a "lo ambiental" y la ausencia de regulación que presenta la actividad, sobre todo en lo que refiere al relacionamiento comunitario. Si bien algunas consultoras son filial de empresas internacionales, por lo que se rigen por ciertos protocolos, la generalidad es que cada una establezca sus propios criterios y metodologías, lo que en la práctica, reconocen, puede traducirse en diferencias significativas en la calidad del trabajo realizado.

Desde el resto de los actores la crítica se endurece al evaluar los compromisos que los expertos han establecido con el mundo privado, sobre todo en el marco de la realización de consultorías. Si bien algunos actores entienden que esto ocurra, observan en esta relación la pérdida total del eje en el que debiera estar centrada la labor universitaria, lo que vinculan, a su vez, con la manera en que el paradigma individualista se ha instalado en la formación.

\section{COMPLEJIDAD DEL PROBLEMA SOCIOAMBIENTAL: MÚLTIPLES OBSERVADORES}

Una de las principales dificultades al enfrentar problemas ambientales se relaciona con la multiplicidad de miradas en torno a ellos. Como lo planteábamos en el comienzo de este artículo, cada observador tiene limita- 
ciones estructurales que se constituyen en puntos ciegos de la observación. De esta forma, los actores relacionados con el ámbito público están limitados por las estructuras de la comunicación política, mientras los actores del ámbito privado, lo están por la comunicación económica y los relacionados con el conocimiento experto están limitados por las estructuras de la comunicación científica.

En la siguiente tabla se presentan las principales distinciones asociadas por los actores de cada ámbito a la comunicación de los sistemas político, jurídico, económico y científico.

TABLA 1. DiSTINCIONES RELACIONADAS CON ÁMBITOS Y DOMINIOS COMUNICATIVOS

\begin{tabular}{|c|c|c|c|}
\hline Comunicación & Ámbito Público & Ámbito Privado & $\begin{array}{l}\text { Ámbito conocimiento } \\
\text { experto }\end{array}$ \\
\hline Política & $\begin{array}{l}\text { Problema ambien- } \\
\text { tal sólo es rele- } \\
\text { vante cuando lo es } \\
\text { también para los } \\
\text { votantes } \\
\text { Problemas am- } \\
\text { bientales como } \\
\text { conflictos sociales }\end{array}$ & $\begin{array}{l}\text { Existencia de conflic- } \\
\text { tos de interés entre } \\
\text { las autoridades polí- } \\
\text { ticas y los empresa- } \\
\text { rios }\end{array}$ & $\begin{array}{l}\text { Poco conocimiento y } \\
\text { entendimiento de las } \\
\text { autoridades y funciona- } \\
\text { rios públicos sobre los } \\
\text { temas ambientales } \\
\text { Falta de coordinación } \\
\text { entre los distintos orga- } \\
\text { nismos con competen- } \\
\text { cia ambiental }\end{array}$ \\
\hline Jurídica & $\begin{array}{l}\text { Debilidad en los } \\
\text { procedimientos y } \\
\text { regulaciones vi- } \\
\text { gentes } \\
\text { Ausencias de } \\
\text { norma y leyes es- } \\
\text { pecíficas nece- } \\
\text { sarias }\end{array}$ & $\begin{array}{l}\text { Necesidad de mejo- } \\
\text { rar la institucionali- } \\
\text { dad ambiental vigen- } \\
\text { te, estableciendo } \\
\text { "reglas claras". }\end{array}$ & $\begin{array}{l}\text { Débiles atribuciones del } \\
\text { Estado, autoridades } \\
\text { tendrían un margen de } \\
\text { acción muy estrecho }\end{array}$ \\
\hline Económica & $\begin{array}{l}\text { Agenda ambiental } \\
\text { estaría supeditada } \\
\text { a la estabilidad } \\
\text { económica }\end{array}$ & $\begin{array}{l}\text { Impactos necesarios } \\
\text { o inevitables debido } \\
\text { a la actividad pro- } \\
\text { ductiva. } \\
\text { Problema socioam- } \\
\text { biental como proble- } \\
\text { ma de rentabilidad. }\end{array}$ & $\begin{array}{l}\text { Preponderancia de los } \\
\text { criterios económicos } \\
\text { por sobre los políticos. }\end{array}$ \\
\hline Científica & $\begin{array}{l}\text { Débil relación en- } \\
\text { tre academia y } \\
\text { comunidades }\end{array}$ & $\begin{array}{l}\text { Falta de generación } \\
\text { de conocimiento } \\
\text { aplicado }\end{array}$ & $\begin{array}{l}\text { Desarrollo de in- } \\
\text { vestigación y gene- } \\
\text { ración de conocimiento. } \\
\text { Principal preocupación: } \\
\text { generación de papers }\end{array}$ \\
\hline
\end{tabular}

FUENTE: Elaboración propia 
A partir de las distinciones aquí identificadas, es posible diferenciar las observaciones que los actores realizan sobre sus propias limitaciones (cuadros grises) y sobre las dificultades que ellos visualizan en los otros ámbitos.

Cuando los actores del ámbito público se refieren a la comunicación política, identifican la relevancia de los conflictos y de la preocupación de los votantes sobre los problemas ambientales. Paralelamente, al referirse al sistema jurídico -que se relaciona estrechamente con sus funcionesreflexionan sobre las limitaciones de la actual institucionalidad.

Por otra parte, cuando los actores relacionados con el mundo privado remiten a su propio ámbito, a la comunicación económica, asocian los problemas ambientales con impactos inevitables de las actividades productivas, identificando además un problema de rentabilidad. Asimismo, la principal demanda o crítica relacionada con el ámbito jurídico refiere a la falta de reglas claras para el desarrollo de actividades productivas, mientras al referirse a la comunicación política cuestionan los conflictos de intereses que existirían entre políticos y empresas. Finalmente, al hacer referencia a la comunicación científica, los actores empresariales cuestionan la falta de generación de conocimiento aplicado.

Por último, en el ámbito del conocimiento experto, cuando se refieren a la comunicación científica, se destaca que lo central es la productividad académica (investigación y papers). Estos expertos, al referirse al ámbito político, se concentran en dos debilidades centrales: la falta de coordinación entre los diferentes organismos y la falta de conocimiento de las autoridades y funcionarios en relación a los temas ambientales. Al referirse a la legislación, indican la falta de atribuciones que tendrían las autoridades para enfrentar los problemas ambientales. Por último, en relación a la comunicación económica, se identifica una clara preponderancia sobre los criterios políticos.

Otra forma de observar las diferencias en las descripciones que realicen los diferentes actores, se relaciona con la identificación del problema ambiental y las expectativas para abordarlo. En la siguiente tabla vemos la comparación de estos elementos. 
TABLA 2. DEFINICIÓN DEL PROBLEMA AMBIENTAL Y EXPECTATIVAS PARA ABORDARLO

\begin{tabular}{|l|l|l|l|}
\hline & Ámbito Público & Ámbito Privado & $\begin{array}{l}\text { Ámbito conocimien- } \\
\text { to experto }\end{array}$ \\
\hline $\begin{array}{l}\text { Problema } \\
\text { Ambiental }\end{array}$ & $\begin{array}{l}\text { Problema técnico que } \\
\text { se origina y resuelve } \\
\text { en el marco de la ins- } \\
\text { titucionalidad am- } \\
\text { biental vigente. }\end{array}$ & $\begin{array}{l}\text { Problema normativo } \\
\text { ocasionado por au- } \\
\text { sencia de "reglas cla- } \\
\text { ras" y normas más } \\
\text { precisas que permi- } \\
\text { tan minimizar costos. }\end{array}$ & $\begin{array}{l}\text { Problema institucional, } \\
\text { relacionado con la } \\
\text { viabilidad de los pro- } \\
\text { yectos y su desarrollo } \\
\text { en el marco de una } \\
\text { institucionalidad } \\
\text { adecuada. }\end{array}$ \\
\hline $\begin{array}{l}\text { Expectativas } \\
\text { para abor- } \\
\text { dar los pro- } \\
\text { blemas am- } \\
\text { bientales }\end{array}$ & $\begin{array}{l}\text { Diferenciación: Nece- } \\
\text { sidad de establecer } \\
\text { límites a la manera en } \\
\text { que lo económico es- } \\
\text { tá influyendo en las } \\
\text { decisiones políticas. }\end{array}$ & $\begin{array}{l}\text { Regulación: Incre- } \\
\text { mento de los están- } \\
\text { dares ambientales } \\
\text { que vienen de la } \\
\text { mano de normativas } \\
\text { y exigencias interna- } \\
\text { cionales. }\end{array}$ & $\begin{array}{l}\text { Politización: Necesi- } \\
\text { dad de que la aca- } \\
\text { demia vuelva a asu- } \\
\text { mir un rol social y } \\
\text { político, más allá de } \\
\text { la mera difusión de } \\
\text { conocimiento. }\end{array}$ \\
\hline
\end{tabular}

FUENTE: Elaboración propia

Es interesante observar cómo, para el ámbito público, el problema ambiental es técnico y se relaciona con la institucionalidad ambiental vigente. Mientras para el ámbito privado, el problema estaría ocasionado por la ausencia de reglas claras, haciendo referencia también a la institucionalidad. Finalmente para el conocimiento experto, el problema también se vincularía con la institucionalidad y la viabilidad de los diferentes proyectos.

$\mathrm{Al}$ hacer referencias a las expectativas de solución o alternativas para abordar estos problemas hay importantes diferencias. Mientras para el ámbito público lo importante es establecer límites a la influencia de lo económico en lo político, para el ámbito privado la clave está en el aumento de los estándares ambientales gracias a las presiones del mercado internacional. Finalmente, en el ámbito del conocimiento experto se identifica la necesidad de que la academia tenga un rol más activo en el ámbito político.

\section{CONCLUSIONES}

$\mathrm{Al}$ analizar los discursos de los representantes de los diferentes ámbitos, es evidente la determinación de las estructuras presentes en los sistemas funcionales con los que se relacionan primordialmente y también de las estructuras organizacionales con las que se vinculan. De esta forma, es 
comprensible que para los funcionarios públicos, cumplir con la norma sea lo más relevante en relación a los problemas ambientales, o que para los empresarios sea posible tematizar estos problemas desde la perspectiva de la rentabilidad.

Por otra parte, se entienden también las críticas que realizan los diferentes actores a otros observadores, donde cada uno está analizando el problema y el actuar de los otros observadores desde sus propias estructuras, sin problematizar los puntos ciegos. Este punto es fundamental, ya que uno de los aspectos claves para avanzar en una coordinación efectiva se relaciona con la capacidad de los sistemas de aprender, a partir de la reflexividad que implica la observación de los propios límites. Si bien en algunos casos los actores problematizan las determinaciones de sus propios ámbitos, no se evidencia una posición clara frente a sus propias estructuras de observación.

Es interesante identificar como -a pesar de las diferencias- hay una mirada general que indica que hay una preponderancia de los criterios económicos por sobre los políticos y que la institucionalidad ambiental no es suficiente para regular los problemas ambientales y para establecer las reglas del juego que permitan desarrollar inversiones.

Finalmente, es importante destacar la ausencia de una mirada a largo plazo que facilite la coordinación entre diferentes perspectivas. Esto es una ausencia importante, ya que precisamente cuando se logre instalar un horizonte temporal mayor, construyendo además una institucionalidad que vele por ello, las evaluaciones económicas se verán obligadas a incorporar los efectos de las diferentes operaciones en el resto de los ámbitos sociales y ambientales, incorporando los costos que ellas implican. Entonces, precisamente gracias a esta mirada a largo plazo existirán mayores posibilidades de lograr el entendimiento entre los diferentes actores.

En definitiva, la investigación antes presentada y discutida nos presenta un panorama paradojal para los problemas socioambientales, esto es, intentar dar cuenta de la unicidad desde la diversidad. Esto no se resuelve simplemente con fórmulas nominales del tipo: "hay muchos ambientes debido a muchos observadores" o "no existe consenso respecto de lo ambiental". El diagnóstico de una sociedad funcionalmente diferenciada muestra en sus fundamentos que estas son condiciones peculiares de su funcionamiento y precaria estabilidad.

El verdadero problema reside más bien en señalar las dinámicas por las cuales cada posición de observación y descripción alcanza niveles de reflexividad que hacen posible llevar sus descripciones al nivel de observa- 
ción de segundo orden y, de este modo, poder al menos mover los puntos ciegos hacia posiciones que los tematicen en la propia discusión. En tanto cada posición sistémica desplace el peso de sus responsabilidades hacia su entorno, no será posible una adecuada articulación de posiciones de manera virtuosa para afrontar los problemas socioambientales. La tarea no es ciertamente sencilla, pero la racionalidad sistémica solamente se alcanza cuando se tolera la introducción del esquema de observación dentro de sí mismo y se maneja la paradoja en su interior. De este modo, lo económico asume que no todo puede ser llevado a sus operaciones, en tanto ellas suponen un estado indiferenciado; lo político recoge la bandera del universalismo desmedido hacia una comunidad política amorfa y resbaladiza y reafirma que la oposición es simplemente un valor de conexión y no un partido o una ideología -ni siquiera un conjunto de todo esto; la ciencia introduce sus propios vacíos de conocimiento como motores de curiosidad hacia lo que acontece más allá de sus fronteras, etc.

La ausencia de una perspectiva global e integradora de la sociedad moderna puede ser no solamente un obstáculo para dar respuestas efectivas a los problemas socioambientales, sino también un terreno fértil para reenfocar estos problemas a la luz de una posición decididamente multifocal. El ambiente de la sociedad se nos presenta precisamente como un punto de reflexión (de introspección y reflejo) donde la sociedad ha de experimentar su propio límite y soportar el vértigo de su propia paradoja. RM

\section{REFERENCIAS}

Douglas, M. (1996). La aceptabilidad del riesgo según las ciencias sociales. Barcelona: Paidós.

Luhmann, N. (1997). Die Gesellschaft der Gesellschaft. Frankfurt aM: Suhrkamp.

Luhmann, N. (1986). Ökologische Kommunikation. Kann die moderne Gesellschaft sich auf ökologische Gefährdungen einstellen? Opladen: Westdeutscher Verlag.

Luhmann, N. (2000). Organisation und Entscheidung. Opladen: Westdeutscher Verlag Spencer-Brown, G. (1979). Laws of Form. New York: EP Dutton.

SOBRE LAS AUTORAS

Anahí Urquiza es Antropóloga social y Magíster en Antropología y Desarrollo por la Universidad de Chile. PhD en Sociología con mención en Geografía por la Universidad Ludwig Maximilian de Múnich, Alemania. PhD en Environment and Society por el Rachel Carson Center de Múnich. Actualmente se desempeña como académica en el Departamento de Antropología de la Universidad de Chile. Además es investigadora del Centro del Clima y la Resiliencia (Cr2) y del Núcleo 
Interdisciplinario de Estudios Socioambientales (NIES), ambos de la Universidad de Chile, e investigadora del proyecto MAPS (Mitigation Action Plans and Scenarios) que convoca a la Universidad de Chile, el Programa de las Naciones Unidas para el Desarrollo (PNUD), la fundación Children Investment Fund (CIFF), la Alianza Clima y Desarrollo (CDKN), y los gobiernos de Suiza, Dinamarca, Chile y Sudáfrica. Entre sus últimas publicaciones se destacan: La organización de las organizaciones sociales (junto a Marcelo Arnold y Hugo Cadenas. RIL, 2014), Niklas Luhmann y el legado universalista de su teoría (junto a Hugo Cadenas y Aldo Mascareño. RIL, 2012), y Medioambiente y sociedad (junto a Enrique Aliste. RIL, 2010).

Bárbara Morales es Antropóloga social y Máster en Sociología por la École des Hautes Études en Sciences Sociales (EHESS) de París, Francia. Candidata a Doctora en Antropología por la misma universidad. Actualmente se desempeña como investigadora del Núcleo Interdisciplinario de Estudios Socioambientales (NIES) y es asistente de investigación del Centro del Clima y la Resiliencia (Cr2), ambos de la Universidad de Chile. Entre sus últimas publicaciones destacan: Defensa del medio ambiente y ciudadanía: etnografía de los procesos de construcción de comunidad en el curso de la acción colectiva (Revista: Persona y sociedad, 2013), La defensa del medioambiente y la construcción de ciudadanía (Revista: Amérique Latine Histoire et Mémoire, 2012) y Tensiones entre lo global y lo local en el conflicto ambiental (junto a Asunción Díaz en "Medio Ambiente y Sociedad" A. Urquiza y E. Aliste (Eds.), Santiago de Chile, 2010).

\section{CONTACTO}

Departamento de Antropología

Facultad de Ciencias Sociales, Universidad de Chile

Avenida Ignacio Carrera Pinto 1045, Ñuñoa

Santiago, Chile

CP: 6850331

anahiurquiza@u.uchile.cl

Recibido: Junio 2015

Aceptado: Agosto 2015 